\title{
Hypophysectomy Increases Vasoactive Intestinal Peptide-Stimulated Cyclic AMP Generation in the Hippocampus of the Rat
}

\author{
Allan L. Harrelson and Bruce S. McEwen \\ Laboratory of Neuroendocrinology, The Rockefeller University, New York, New York 10021
}

In investigating the feedback effects of circulating hormones on the brain, we showed previously that adrenalectomy (ADX) increases vasoactive intestinal peptide (VIP)-stimulated cAMP generation in slices from rat hippocampus, a brain structure with high levels of glucocorticoid receptors. This effect is reversed by replacement with glucocorticoids such as dexamethasone (DEX) and corticosterone (CORT). Here we report that, like ADX, hypophysectomy (HYPOX) also elevates VIP-stimulated CAMP generation, compared with sham-operated controls. Moreover, like glucocorticoid replacement, administration of ACTH to HYPOX rats causes a decrease in CAMP stimulation by VIP. Furthermore, ACTH had no effect when given to HYPOX + ADX rats, indicating that the effects of $A C T H$ require the presence of adrenal steroid secretion. However, we find that ACTH may have a permissive role in this glucocorticoid effect because, in the absence of the pituitary, DEX treatment does not decrease VIP-stimulated cAMP levels in the hippocampus. In addition, hippocampal $\beta$-adrenergic-stimulated CAMP accumulation was not suppressed by DEX treatment of HYPOX rats, which again is different from the effect of DEX treatment on ADX animals. These results are discussed in terms of possible synergism between pituitary hormones and steroid hormones in exerting feedback actions on brain function.

The nervous system controls the endocrine response to stressful stimuli and is also a target for the actions of adrenal steroids and ACTH secreted in stress (McEwen et al., 1986). The feedback actions of adrenal steroids are mediated, at least in part, via intracellular receptors and lead to neurochemical and structural alterations in the nervous system (McEwcn, 1982; McEwcn et al., 1986; Sapolsky et al., 1986). We have shown previously that in vivo glucocorticoid treatment influences the ability of several putative neurotransmitters to stimulate cAMP accumulation in slices from rat hippocampus (Harrelson et al., 1987), a brain region containing high levels of specific receptors for glucocorticoids (McEwen et al., 1968; Gerlach and McEwen, 1972; Sapolsky et al., 1983). Since these effects occurred only when glucocorticoid levels were manipulated in vivo, it is possible that this steroid action on the hippocampus might be mediated by other hormones, e.g., by pituitary hormones such as $\mathrm{ACTH}$, which is regulated by glucocorticoids.

A number of investigators have reported that pituitary hor-

Received Sept. 29, 1986; revised Feb. 25, 1987; accepted Mar. 24, 1987.

This work was supported by NIMH Grant MH 41256 to B.S.M.

Correspondence should be addressed to Dr. Harrelson at Department of Biological Sciences, Stanford University, Stanford, CA 94305.

Copyright (C) 1987 Society for Neuroscience $0270-6474 / 87 / 092807-04 \$ 02.00 / 0$ mones, including ACTH, can alter the function of the CNS, including effects on memory, electrophysiology, and neurochemistry (Pfaff et al., 1971; Urban and de Weid, 1976; Kendall et al., 1982; Olpe and Jones, 1982; Veldhuis and de Kloet, 1982; Markey and Sze, 1984). In order to test the hypothesis that our previous results might involve the action of pituitary hormones such as ACTH on the nervous system, we measured neurotransmitter-stimulated cAMP accumulation in hippocampal slices from hypophysectomized (HYPOX) rats that had been treated in various ways. Our results suggest that glucocorticoids act directly on the brain to cause changes in CAMP synthesis but that ACTH may have a permissive role in this steroid action.

\section{Materials and Methods}

Materials. ACTH was obtained as Acthar Gel from Armour Pharmaceuticals. Purified porcine VIP was obtained from Dr. Viktor Mutt (Stockholm). Rolipram (ZK 62,711) was a gift from Schering AG (Berlin). Pellets of $25 \%$ dexamethasone (DEX) were prepared according to Meyer et al. (1979). Average implant weight was $145 \mathrm{mg}$.

Animals, surgery, and steroid treatment. HYPOX male rats were purchased from Charles River Breeding Co. and delivered $7 \mathrm{~d}$ after surgery The rats were 8-12 weeks old at the beginning of each experiment and weighed approximately $180-240 \mathrm{gm}$. The animals were housed on a 14: 10 light : dark schedule with lights on at 5 a.m. Food (Purina Lab Chow for rats) and water were available ad libitum. HYPOX rats were maintained on 5\% dextrose until being sacrificed 2-3 weeks later. Where indicated, HYPOX rats were injected with 8 units/d ACTH. Adrenalectomies (ADX) were bilateral via a dorsal approach. Animals were tested $3 \mathrm{~d}$ after ADX. In sham-operated animals, incisions were made in the body wall and the adrenals were sighted but not removed. ADX rats were given $0.9 \% \mathrm{NaCl}$ in drinking water to maintain salt balance.

Slice preparation, stimulation, and $C A M P$ measurement. Rats were killed by decapitation. The brains were rapidly removed and dissected on ice (Rotsztejn et al., 1980). Each dissected hippocampus was sliced on a McIlwain tissue chopper twice at right angles at a thickness of $0.325 \mathrm{~mm}$. Sliced hippocampal tissue was immediately transferred to Erlenmeyer flasks filled with oxygenated Krebs-Ringer buffer (concentrations in mM: NaCl, 124; KCl, 5.00; $\mathrm{NaHCO} 3,26.0 ; \mathrm{CaCl}_{2}, 0.800$; $\mathrm{MgCl}_{2}, 1.30 ; \mathrm{KH}_{2} \mathrm{PO}_{4}, 1.40$; dextrose, $10.0, \mathrm{pH} 7.4$ ). The volume of buffer was adjusted to give a wet-weight: volume ratio of $1: 100$. The slices were rinsed with buffer once, and the flasks were then flushed with oxygen, capped, and placed in a shaking water bath at $37^{\circ} \mathrm{C}$ at a moderate shaking speed ( 150 rotations $/ \mathrm{min}$ ). The preincubation period lasted for $60 \mathrm{~min}$; each flask was flushed with $95 \%$ oxygen $/ 5 \%$ carbon dioxide every $10 \mathrm{~min}$, and the medium was aspirated and replaced with an equal volume of fresh Krebs-Ringer medium at 20 and $40 \mathrm{~min}$. For the last $40 \mathrm{~min}$ of preincubation, the medium contained $30 \mu \mathrm{M}$ Rolipram (ZK 62,711), a phosphodiesterase inhibitor (Schwabe, 1976).

At the end of the preincubation period, slices were pipetted from the Erlenmeyer flasks into $1.5 \mathrm{ml}$ plastic minivials, $450 \mu \mathrm{l}$ each. The vials were then flushed with oxygen and tightly capped and placed into horizontal carriers in the shaking water bath. After $10 \mathrm{~min}$, the vials were removed from the bath and $50 \mu \mathrm{l}$ of neurotransmitter stock solution was added; the vials were once again flushed with oxygen, capped tightly, and placed back into the shaking water bath. Twelve minutes later they were removed, the medium quickly aspirated from the slices, and 250 


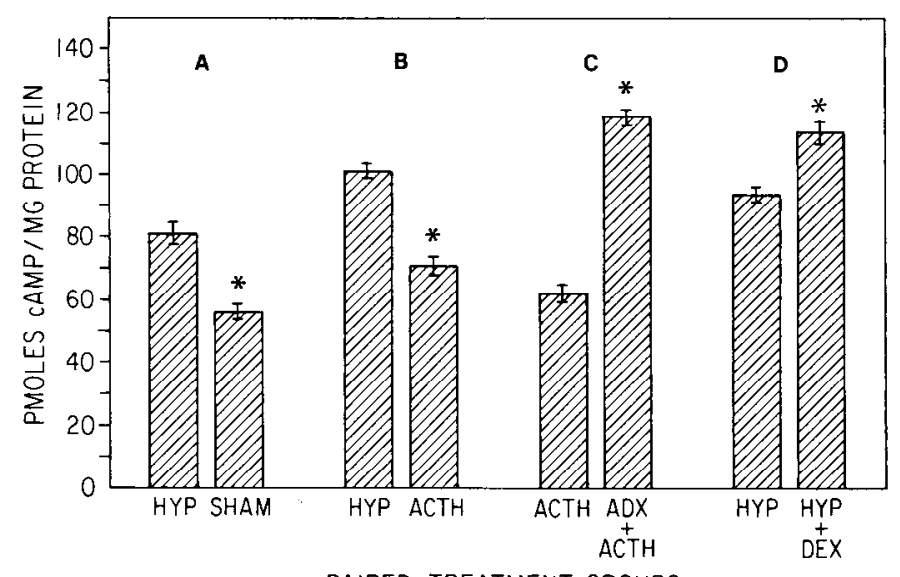

PAIRED TREATMENT GROUPS

Figure 1. VIP-stimulated cAMP accumulation in hippocampal slices: Effect of HYPOX and replacement with DEX and ACTH. VIP concentration, $50 \mathrm{nM}$. SHAM, sham hypophysectomized; $H Y P$, hypophysectomized; $I I Y P+D E X$, hypophysectomized and replaced with $25 \%$ DEX implants; $A C T H$, hypophysectomized and replaced with daily injection of 8 units of ACTH for $6 \mathrm{~d} ; A D X+A C T H$, hypophysectomized and adrenalectomized and replaced with 8 units of ACTH. Experiments were done as paired groups (e.g., 5 separate experiments, each with one HYP and one SHAM rat). Each bar represents the mean \pm SEM stimulation over basal level of 19-30 determinations in 5-8 animals. $A s$ terisks indicate significant differences between paired groups at $p<0.05$ [Duncan's multiple range test following 2-way analysis of variance (ANOVA)].

$\mu \mathrm{l}$ of ice-cold $7 \%$ trichloroacetic acid (TCA) added to each tube. The cAMP generation is linear with time up to $20 \mathrm{~min}$ and with protein amount (data not shown).

The TCA-treated slices were sonified and then centrifuged for $10 \mathrm{~min}$ in a Beckman microfuge at $10,000 \times \mathrm{g}$. The pellets were resuspended in $250 \mu$ of $0.2 \mathrm{~N} \mathrm{NaOH}$ by sonification and assayed for protein by the procedure of Bradford (1976). The TCA supernatants $(250 \mu \mathrm{l})$ were extracted 3 times with 6 volumes of diethyl ether and placed in a $37^{\circ} \mathrm{C}$ water bath to evaporate any remaining traces of ether. After 90 min the supernatants were removed from the water bath, and $10 \mu \mathrm{l}$ of $\mathrm{pH} 7.4$ Tris $(750 \mathrm{~mm})$ was added to each, to neutralize any leftover TCA and correct the $\mathrm{pH}$. Aliquots of supernatant were then taken for cAMP assay by the method of Brown et al. (1971).

The results of all experiments were subjected to analyses of variance and where significant differences occurred were tested with the Student's $t$ test for planned pair comparisons or with the Duncan's test for post hoc comparisons of multiple groups.

\section{Results}

Because DEX decreases VIP-stimulated cAMP accumulation in the hippocampus of ADX rats (Harrelson et al., 1987), we first tested the effects of DEX replacement on HY POX animals, expecting that if glucocorticoids were acting directly on the CNS, then DEX would producc the same cffect that occurs in ADX animals. Unexpectedly DEX did not reduce VIP stimulation in HYPOX animals; instead, there was a slight increase (Fig. $1 D$ ). We obtained the same paradoxical effect when we measured $\beta$-adrenergic stimulation of hippocampal cAMP accumulation in DEX-treated HYPOX rats (Table 1). In this case, glucocorticoid replacement caused isoproterenol (ISOP)- and norepinephrine (NE)-stimulated cAMP levels to go up (Table 1), not down, as was the case in ADX animals (Roberts et al., 1984; Harrelson et al., 1987).

These results imply that $\mathrm{ACTH}$, or some other pituitary hormone, might be involved in the effects of DEX. However, if glucocorticoids have the predominant influence on VIP-stimulated cAMP accumulation, then HYPOX, resulting in loss of
Table 1. Stimulation of cAMP accumulation in hippocampal slices of HYPOX rats by isoproterenol and norepinephrine

\begin{tabular}{lll} 
Treatment group & $\begin{array}{l}\text { Isoproterenol, } \\
50 \mu \mathrm{M}\end{array}$ & $\begin{array}{l}\text { Norepinephrine, } \\
100 \mu \mathrm{M}\end{array}$ \\
\hline HYPOX & $38.1 \pm 4.1$ & $85.6 \pm 5.7$ \\
& $(10)$ & $(15)$ \\
HYPOX + DEX & $56.4 \pm 5.2^{*}$ & $108.0 \pm 9.7^{*}$
\end{tabular}

Isoproterenol- and norepinephrine-stimulated cAMP accumulation in hippocampal slices from HYPOX and HYPOX $+25 \%$ DEX pellet-implanted rats. Each value represents the mean \pm SEM stimulation over basal level. Asterisks indicate significant differences at $p<0.05$ [Duncan's multiple range test following 2-way analysis of variance (ANOVA)] HYPOX versus HYPOX + DEX.

anterior pituitary ACTH secretion, would abolish glucocorticoid secretion from the adrenal gland and produce an effect similar to ADX. That is exactly what occurred (Fig. $1 A$ ): i.e., 2 weeks after HYPOX, hippocampal VIP-stimulated cAMP generation was increased compared with sham-operated controls. Conversely, when we gave ACTH injections to HYPOX rats for 6 $\mathrm{d}$ and compared their hippocampal VIP stimulation of CAMP with that of vehicle-injected HYPOX controls, the ACTH treatment caused a reduction (Fig. $1 B$ ). This is what would be expected if adrenal glucocorticoid stimulation brought about by ACTH was primarily responsible.

It is nevertheless possible that regulation of the cAMP response to VIP was brought about, at least in part, by a direct action of the injected ACTH on the brain. We next determined whether the primary ACTH action was on the brain or on the adrenal gland by comparing the response to ACTH of HYPOX and HYPOX + ADX rats. As shown in Figure $1 C$, the HYPOX rats without adrenals, and hence without the ability to secrete glucocorticoids in response to $\mathrm{ACTH}$, do not respond to the ACTH treatment. Thus, the adrenal gland seems to be required for ACTH to act.

\section{Discussion}

Our results on VIP-stimulated cAMP generation in the hippocampus of HYPOX rats are summarized in Table 2, along with the results of our previous studies on ADX animals (Harrelson et al., 1987). There is an inverse correlation between the changes in glucocorticoid levels and VIP stimulation in the hippocampus, i.e., when corticosterone (CORT) levels go down, cAMP generation goes up; and when CORT levels increase, cAMP accumulation goes down. In contrast, there is no correlation between expected $\mathrm{ACI} H$ levels and $\mathrm{VIP}$-stimulated cAMP accumulation. This implies that adrenal glucocorticoids, not ACTH, are the principal rcgulators of hippocampal cAMP accumulation and that these steroids act directly on the CNS, and not on the pituitary gland, to produce these effects. DEX appears to have the same effect as CORT, the natural glucocorticoid, as long as ACTH is present, and this is consistent with the involvement of the so-called Type 2 glucocorticoid receptor, which binds CORT as well as DEX (McEwen et al., 1986).

There are cases in which the effects of drugs and nonsteroidal hormones on the CNS do not occur in HYPOX animals (Kikuyama et al., 1979; Heal et al., 1983). For example, thyroid hormone has profound effects on brain development in a variety of species, and it has been found that the effects of this hormone on thyroid-deficient tadpoles are blocked by HYPOX (Kiku- 
yama et al., 1979). There are also instances in which the effects of HYPOX on CNS biochemistry are not reversed by DEX (Lando and Raynaud, 1980; Markey and Sze, 1984) or are only partially reversed (Wong et al., 1982). Moreover, ACTH or its metabolites can have effects independent of the adrenals, e.g., the ability of HYPOX to decrease GABA receptors in midbrain cannot be reversed by corticosterone administration, whereas replacement with a nonsteroidogenic ACTH fragment is effective in HYPOX rats (Kendall et al., 1982). Apparently, in the present system, ACTH plays a permissive role in the glucocorticoid-induced changes involving VIP, in that ACTH must be present in some minimal quantity for the glucocorticoids to exert their suppressive effect on cAMP generating capacity (see further discussion below).

Knowledge of how ACTH might act on the nervous system is limited. Peripheral injections of ACTH affect the hippocampus and other regions of the CNS. Pfaff et al. (1971) found that i.v. administration of ACTH to rats caused significant changes in hippocampal unit activity. A similar result on the hippocampal EEG was found by Urban and de Wied (1976). Furthermore, microiontophoretic application of ACTH to the locus coeruleus has excitatory effects, and peripheral injections of the noncorticotrophic fragment, $\mathrm{ACTH}(4-10)$, facilitate receptor responses in the frontal cortex to antidepressant drugs and increase tyrosine hydroxylase activity in the locus coeruleus (Kendall et al., 1982; Olpe and Jones, 1982; Markey and Sze, 1984).

There is little indication as to how circulating ACTH might get to the hippocampus since peripheral ACTH does not cross the blood-brain barrier in large amounts (Krieger and Liotta, 1979), although it has been reported that blood-borne ACTH binds specifically to the median eminence-arcuate region of the rat hypothalamus (Van Houten et al., 1981). Alternatively, the action of ACTH might be mediated by the PNS. Recent studies on the sympathetic innervation of the rabbit pulmonary artery and the rat heart, as well as $\alpha$-motoneurons in the frog, have discovered presynaptic ACTH receptors that, when activated, potentiate the release of neurotransmitters from these terminals (Bassett et al., 1978; Gothert, 1981, 1984; Johnston et al., 1983). Earlier investigations demonstrated that ACTH decreased transmitter release in rat phrenic nerve-diaphragm preparations (Birnberger et al., 1977).

Whether by direct actions on the hippocampus or indirect action through other neural structures in the CNS or periphery, $\mathrm{ACTH}$ is required for the suppressive effects of glucocorticoids on VIP-stimulated cAMP accumulation. Moreover, ACTH appears to determine the direction of this glucocorticoid effect since in the absence of the pituitary, DEX tends to increase cAMP generating capacity, whereas in the presence of the pituitary, DEX decreases it. The positive effect of DEX has further possible biological significance in that female rats, with their pituitary glands in place, respond to DEX likc HYPOX males by increasing cAMP generating capacity (Harrelson and McEwen, 1987).

While the importance of this requirement for ACTH in the overall control of the stress response by the nervous system can only be a subject for speculation at this point, there is nevertheless growing interest in the question of synergism between piluilary and steroid hormones as a mechanism in hormone feedback on the brain. For example, in order for estradiol to decrease hypothalamic VIP content in female rats, high levels of serum prolactin are required, even though prolactin by itself has no effect (Maletti et al., 1982). Moreover, in another instance
Table 2. Pituitary-adrenal steroid regulation of hippocampal VIPstimulated cAMP

\begin{tabular}{llll} 
& & & $\begin{array}{l}\text { Hippocampal } \\
\text { VIP- } \\
\text { stimulated }\end{array}$ \\
Endocrine status & CORT & ACTH & cAMP \\
\hline ADX & - & + & Increased \\
ADX + CORT & + & - & Decreased \\
HYPOX & & - & Increased \\
HYPOX + ACTH & + & + & Decreased \\
HYPOX + ACTH + ADX & - & + & Increased \\
\hline
\end{tabular}

Regulation of VIP-stimulated cAMP accumulation in hippocampal slices by alterations in the pituitary-adrenal axis. - , absent or extremely low; + , present at normal or high levels.

where a steroid hormone alters a neurotransmitter-related event, it has recently been reported that an as-yet-unidentified pituitary factor is required for estradiol to decrease transcription of the gene for tyrosine hydroxylase in the rat hypothalamus (Blum et al., 1986, 1987). In the absence of the pituitary, estradiol increases tyrosine hydroxylase gene transcription (Blum et al., 1986). Similarly, the present study has shown that DEX treatment in the absence of circulating ACTH increases the cAMP generation elicited by VIP. In ADX rats, where ACTH is present, DEX treatment decreases VIP-stimulated cAMP synthesis (Harrelson et al., 1987). Thus, in both cases, the additional pituitary factor is a "switch" that changes the direction of the steroid effect. These examples are presently without a molecular explanation, but they serve to raise the possibility that interactions between steroid and pituitary hormones may occur frequently in the nervous system and contribute to the multifaceted endocrine influences on the brain that affect behavior as well as neuroendocrine output.

\section{References}

Bassett, J. R., F. L. Strand, and K. D. Cairncross (1978) Glucocorticoids, adrenocorticotropic hormone, and related polypeptides on myocardial sensitivity to noradrenaline. Eur. J. Pharmacol. 49: 243249.

Birnberger, K. L., R. Rudel, and A. Struppler (1977) ACTH and neuromuscular transmission: Electrophysiological in vitro investigation of the effects of corticotropin and an ACTH fragment on neuromuscular transmission. Ann. Neurol. 1: 270-275.

Blum, M., B. S. McEwen, and J. L. Roberts (1986) Regulation of TH gene transcription by estrogen in the arcuate nucleus of the female rat. Soc. Neurosci. Abstr. 12: 102.10.

Blum, M., B. S. McEwen, and J. L. Roberts (1987) Transcriptional analysis of tyrosine hydroxylase gene expression in the tuberoinfundibular dopaminergic neurons of the rat arcuate nucleus after estrogen treatment. J. Biol. Chem. 262: 817-821.

Bradford, M. (1976) A rapid and sensitive method for the quantitation of microgram quantities of protein utilizing the principle of proteindye binding. Anal. Biochem. 72: 248-254.

Brown, B. L., J. D. M. Albano, R. P. Ekins, A. M. Sgherzi, and W. Pampion (1971) A simple and sensitive saturation assay method for the measurement of adenosine $3^{\prime}: 5^{\prime}$-cyclic monophosphate. Biochem. J. 121: 561-562.

Gerlach, J. L., and B. S. McEwen (1972) Rat brain binds adrenal steroid hormone: Radioautography of hippocampus with corticosterone. Science 175: 1133-1136.

Gothert, M. (1981) ACTH(1-24) increases stimulation-evoked noradrenaline release from sympathetic nerves by acting on presynaptic ACTH receptors. Eur. J. Pharmacol. 76: 295-296.

Gothert, M. (1984) Facilitatory effect of adrenocorticotropic hormone and related peptides on $\mathrm{Ca}\left(2^{+}\right)$-dependent noradrenaline release from sympathetic nerves. Neuroscience 11: 1001-1009. 
Harrelson, A. L., and B. S. McEwen (1987) Gonadal steroid modulation of neurotransmitter-stimulated cAMP accumulation in the hippocampus of the rat. Brain Res. 404: 89-94.

Harrelson, A. L., W. Rostene, and B. S. McEwen (1987) Adrenocortical steroids modify neurotransmitter-stimulated cAMP accumulation in the hippocampus and limbic brain of the rat. J. Neurochem. 48: $1648-1655$.

Heal, D. J., K. M. O'Shaughnessy, S. L. Smith, and D. J. Nutt (1983) Hypophysectomy alters both 5-hydroxytryptamine and alpha(2)-adrenoceptor-mediated behavioural changes in the rat. Eur. J. Pharmacol. 89: 167-171.

Johnston, M. F., E. A. Kravitz, H. Meiri, and R. Rahamimoff (1983) Adrenocorticotropic hormone causes long-lasting potentiation of transmitter release from frog motor nerve terminals. Science 220 : 1071-1072.

Keller-Wood, M. E., and M. F. Dallman (1984) Corticosteroid inhibition of ACTH secretion. Endocrine Rev. 5: 1-24.

Kendall, D. A., B. S. McEwen, and S. J. Enna (1982) The influence of ACTH and corticosterone on $\left[{ }^{3} \mathrm{H}\right] \mathrm{GABA}$ receptor binding in rat brain. Brain Res. 236: 365-374.

Kikuyama, S., M. Miyakawa, and Y. Arai (1979) Influence of thyroid hormone on the development of preoptic-hypothalamic monoaminergic neurons in tadpoles of Bufo bofo japonicus. Cell Tissue Res. 198: 27-33.

Krieger, D. T., and A. S. Liotta (1979) Pituitary hormones in brain: Where, how and why? Science 205: 366-372.

Lando, D., and J. P. Raynaud (1980) Adrenocorticotropin analogs and glucocorticoids in the hypophysectomized rat. II. Effects on cerebral cortex polyribosomes. Endocrinology 107: 2063-2068.

Maletti, M., W. Rostene, L. Carr, H. Scherrer, D. Rotten, C. Korkon, and G. Rosselin (1982) Interaction between estradiol and prolactin on vasoactive intestinal peptide concentrations in the hypothalamus and in the anterior pituitary of the female rat. Neurosci. Lett. 32: 307-313.

Markey, K. A., and P. Y. Sze (1984) Influence of ACTH on tryosine hydroxylase activity in the locus coeruleus of mouse brain. Neuroendocrinology 38: 269-275.

McEwen, B. S. (1982) Glucocorticoids and hippocampus; receptors in search of a function. In Current Topics in Neuroendocrinology: Adrenal Actions on Brain, D. Gantner and D. Pfarr, eds., pp. 1-22, Springer-Verlag, Berlin.

McEwen, B. S., J. M. Weiss, and L. S. Schwartz (1968) Selective retention of corticosterone by limbic structures in rat brain. Nature 220: 911-912.

McEwen, B. S., E. R. de Kloet, and W. Rostene (1986) Adrenal steroid receptors and actions in the nervous system. Physiol. Rev. 66: 11211188.
Meyer, J. S., D. J. Micco, B. S. Stephenson, L. C. Krey, and B. S. McEwen (1979) Subcutaneous implantation method for chronic glucocorticoid replacement therapy. Physiol. Behav. 22:867-870.

Mobley, P. L., H. Mannier, and F. Sulser (1983) Norepinephrinesensitive adenylate cyclase system in rat brain: Role of adrenal corticosteroids. J. Pharm. Exp. Ther. 226: 71-77.

Olpe, H. R., and R. S. G. Jones (1982) Excitatory effects of ACTH on nonadrenergic neurons of the locus coeruleus in the rat. Brain Res. 251: $177-179$

Pfaff, D. W., M. T. A. Silva, and J. M. Weiss (1971) Telemetric recording of hormone effects on hippocampal neurons. Science 172: 394-395.

Roberts, V. J., R. L. Singhal, and D. C. S. Roberts (1984) Corticosterone prevents the increase in noradrenaline-stimulated adenyl cyclase activity in rat hippocampus following adrenalectomy or metopirone. Eur. J. Pharmacol. 103: 235-240.

Rotsztejn, W. H., J. Besson, B. Briaud, L. Gagnant, G. Rosselin, and C. Cordon (1980) Effect of steroids on vasoactive intestinal peptide in discrete brain regions and peripheral tissues. Neuroendocrinology 31: $282-286$

Sapolsky, R. M., B. S. McEwen, and T. C. Rainbow (1983) Quantitative autoradiography of $\left[{ }^{3} \mathrm{H}\right]$ corticosterone receptors in rat brain. Brain Res. 271: 331-334.

Sapolsky, R. M., L. C. Krey, and B. S. McEwen (1986) The neuroendocrinology of stress and aging: The glucocorticoid cascade hypothesis. Endocrine Rev. 7: 284-301.

Schwabe, U., M. Miyake, Y. Ohga, and J. W. Daly (1976) 4-(3-Cyclopentyloxy-4-methoxyphenyl)-2-pyrrolidone (ZK 62711): Potent inhibitor of adenosine cyclic $3^{\prime}, 5^{\prime}$-monophosphate phosphodiesterases in homogenates and tissue slices from rat brain. Mol. Pharmacol. 12: 900-910.

Urban, I., and D. de Wied (1976) Changes in excitability of the theta activity generating substrate by ACTH 4-10 in the rat. Exp. Brain Res. 24: 325-334

Van Houten, M., M. N. Kahn, R. J. Kahn, and B. I. Posner (1981) Blood-borne adrenocorticotropin binds specifically to the median eminence-arcuate region of the rat hypothalamus. Endocrinology 108 : 2385-2387.

Veldhuis, H. D., and E. R. de Kloet (1982) Vasopressin-related peptides increase the hippocampal corticosterone receptor capacity of diabetes insipidus (Brattleboro) rats. Endocrinology 110: 153-157.

Wong, D. L., E. L. Zager, and R. D. Ciaranello (1982) Effects of hypophysectomy and dexamethasone administration on central and peripheral S-adenosylmethionine levels. J. Neurosci. 2: 758-764. 